\title{
Effect of electro-acupuncture on the BDNF-TrkB pathway in the spinal cord of CCI rats
}

\author{
WEN-ZHAN TU ${ }^{1,2,5^{*}}$, SI-SI LI ${ }^{2 *}$, XIA JIANG ${ }^{2}$, XIN-RU QIAN ${ }^{3,5}$, GUAN-HU YANG $^{4,5}$, \\ PENG-PENG GU ${ }^{2}$, BIN LU ${ }^{6}$ and SONG-HE JIANG ${ }^{2,5}$
}

\begin{abstract}
${ }^{1}$ Shandong University, Jinan, Shandong 250100; ${ }^{2}$ Department of Physical Medicine and Rehabilitation, The Second Affiliated Hospital and Yuying Children's Hospital of Wenzhou Medical University, Wenzhou, Zhejiang 325027, P.R. China; ${ }^{3}$ Department of Rehabilitation, NYU Langone Medical Center, New York, NY 10016; ${ }^{4}$ Department of Specialty

Medicine, Ohio University, Athens, OH 45701, USA; ${ }^{5}$ Integrative \& Optimized Medicine Research Center, China-USA Institute for Acupuncture and Rehabilitation of Wenzhou Medical University, Wenzhou, Zhejiang 325027;

${ }^{6}$ Protein Quality Control and Diseases Laboratory, Attardi Institute of Mitochondrial Biomedicine, School of Life Sciences, Wenzhou Medical University, Wenzhou, Zhejiang 325035, P.R. China
\end{abstract}

Received August 8, 2016; Accepted January 23, 2018

DOI: $10.3892 / \mathrm{ijmm} .2018 .3563$

\begin{abstract}
Microglia, which comprise a sensor for pathological events in the central nervous system, may be triggered by nerve injury and transformed from a quiescent state into an activated state; ionised calcium binding adaptor molecule 1 (Iba1) is a sensitive marker associated with activated microglia. Accumulated evidence suggests that spinal activated microglia and the brain-derived neurotrophic factor (BDNF)-tyrosine kinase receptor $B$ (TrkB) signalling pathway play major roles in the production and development of neuropathic pain. Electro-acupuncture (EA) has a positive effect on relieving chronic neuropathic pain; however, the underlying mechanisms remain unclear. To determine the significance of EA in the treatment of neuropathic pain mediated by activated microglia and the BDNF-TrkB signalling pathway in the spinal cord, the mechanical withdrawal threshold (MWT) and thermal withdrawal latency (TWL) values were recorded to assess hyperalgesia and allodynia. In addition, the amount of activated microglia and BDNF were assessed via immunofluorescence.
\end{abstract}

Correspondence to: Professor Song-He Jiang, Department of Physical Medicine and Rehabilitation, The Second Affiliated Hospital and Yuying Children's Hospital of Wenzhou Medical University, 268 Xueyuanxi Road, Wenzhou, Zhejiang 325027, P.R. China

E-mail: songhe.jiang@gmail.com

Professor Bin Lu, Protein Quality Control and Diseases Laboratory, Attardi Institute of Mitochondrial Biomedicine, School of Life Sciences, Wenzhou Medical University, University Town, Chashan, Wenzhou, Zhejiang 325035, P.R. China

E-mail: lubmito@wmu.edu.cn

*Contributed equally

Key words: electro-acupuncture, brain-derived neurotrophic factor, tyrosine kinase receptor $\mathrm{B}$, spinal cord, chronic constriction injury
Iba1, BDNF and TrkB mRNA expression levels were examined using qPCR; the protein levels of BDNF, p-TrkB and TrkB in the spinal cord were analysed via western blotting. The present study demonstrated that EA treatment increased the MWT and TWL values. EA significantly inhibited the proportion of activated microglia and BDNF expression in the spinal cord after chronic constrictive injury (CCI). Furthermore, EA decreased the expression of BDNF and TrkB at both the mRNA and protein levels in the spinal cord of CCI rats. These findings suggest that the analgesic effect of EA may be achieved by inhibiting the activation of spinal microglia and subsequently blocking the BDNF-TrkB signalling pathway.

\section{Introduction}

Neuropathic pain syndromes, i.e., pain after a lesion or disease of the peripheral or central nervous system (CNS), are clinically characterised by spontaneous pain (ongoing pain, paroxysms) and evoked types of pain (hyperalgesia, allodynia) (1). Previous studies have focused on the relationship between the neurotransmitters involved in neuropathic pain and modulators such as serotonin, opioids and adenosine (2-4). Recently, increasing evidence indicates that spinal microglia contribute to the establishment and maintenance of central sensitisation in chronic neuropathic pain (5). The activation of microglia stimulates the synthesis and release of brain-derived neurotrophic factor (BDNF) (6), which modulates neuronal excitability within the spinal cord and plays an indispensable role in central sensitisation (7). BDNF mediates its action through various intracellular signalling pathways triggered by the activation of tyrosine kinase receptor B (TrkB) (8). Electroacupuncture (EA) is widely accepted as a viable therapeutic intervention for chronic pain treatment $(9,10)$; however, the biological basis and the precise mechanism that underlies the analgesic effect of EA remain unknown. In traditional Chinese medicine, the Zusanli (ST-36) acupoint of the 'Stomach Meridian of Foot-Yangming' and the Yanglingquan (GB-34) 
acupoint of the 'Gall Bladder Meridian of Foot-Shaoyang' are commonly used to treat neuropathic pain $(11,12)$. The aim of this study was to investigate whether the analgesic effect of EA is associated with the following mechanisms: i) the inhibition of the activation of spinal microglia; ii) the disruption of the BDNF-TrkB signalling pathway.

\section{Materials and methods}

Animals. The Institutional Animal Care and Use Committee of Wenzhou Medical University approved all experiments, which were performed according to the guidelines of the National Institutes of Health Guide for the Care and Use of Laboratory Animals (NIH Publications no. 80-23, revised 1978). All efforts were made to minimise the number of animals used and their suffering. Male Sprague Dawley rats, which weighed 200-250 g, were used in this study. All animals were housed in plastic boxes at a temperature of $22-24^{\circ} \mathrm{C}$ and were provided ad libitum food and water under a $12 \mathrm{~h}$ reversed light-dark cycle.

Chronic constriction injury model. As the most commonly used animal model of neuropathic pain, the chronic constriction injury (CCI) model, which was generated based on previously described methods, was implemented to investigate the questions asked in this study (13). Briefly, male rats were anaesthetised with $4 \%$ chloral hydrate $(10 \mathrm{ml} / \mathrm{kg}$, i.p.); the adequacy of the anaesthesia was verified based on a lack of response to a nociceptive stimulus. The right sciatic nerve was exposed at the mid-thigh level and approached to the sciatic trifurcation; four ligature knots (4-0 chromic gut) were loosely tied, with $\sim 1 \mathrm{~mm}$ between the knots. The knots slightly constricted the nerve; however, they did not interrupt the circulation through the epineural vasculature. For the rats in the sham CCI group, the sciatic nerve was exposed for 2-3 min without ligatures. The rats were randomly divided into 4 groups, including the normal group, sham CCI group, CCI group and CCI plus EA group. There were 15 rats/group. The mechanical withdrawal threshold (MWT) and the thermal withdrawal latency (TWL) of every rat were measured as an assessment of nociception prior to the CCI operation and at days 3, 5, 7, 10, 12 and 14 after the CCI operation. The behavioural testing was conducted between 14:00 and 18:00.

MWT. The MWT was measured using a 2392 Electronic von Frey Anesthesiometer (IITC Life Science, Woodland Hills, CA, USA) to evaluate mechanical allodynia. The animals were individually placed inside wire-mesh-bottom cages $(20 \times 14 \times 16 \mathrm{~cm})$ and allowed to adapt for $30 \mathrm{~min}$ prior to testing. The plantar surface of the paw was stimulated with a series of ascending von Frey filaments that ranged from 0.1 to $70 \mathrm{~g}$ until the rat twitched its paw. The maximum force was recorded at which the animal briskly lifted its hind paw. Each rat was tested 6 times at intervals of $5 \mathrm{~min}$, and the average value was used as the MWT.

TWL. A 37370 plantar test apparatus (Ugo-Basile, Milan, Italy) was used to measure the TWL to estimate thermal hyperalgesia. The rats were placed in a transparent, square, bottomless acrylic box $(17 \times 11.5 \times 14 \mathrm{~cm})$. After $15 \mathrm{~min}$, the radiant heat was set to $60^{\circ} \mathrm{C}$ and was applied via a direct beam of light to the foot pad of each hind paw through a glass plate. The light beam turned off automatically when the rat lifted its paw. The cut-off time for the heat stimulation was $40 \mathrm{sec}$. Each hind paw was alternately tested at 10 min intervals. Each rat was tested 5 times, and the average value for the withdrawal time was used as the TWL.

EA stimulation. One needle was placed at the Zusanli acupoint (ST-36), which was located $5 \mathrm{~mm}$ beneath the capitulum fibulae and lateral posterior to the knee-joint, and another needle was placed at the Yanglingquan acupoint (GB-34), which was located on the gall bladder meridian and was $\sim 5 \mathrm{~mm}$ superior-lateral to ST-36 (14,15) (Fig. 1). The rats were allowed to recover for 7 days; EA started on the eighth day after the CCI surgery and lasted for 7 days. EA was performed between 09:00 and 11:00 every day. The rats were restrained in an immobilisation apparatus designed by our laboratory (patent application no. 201110021482.5; State Intellectual Property Office) without anaesthesia; the apparatus is convenient for acupuncture research and comfortable for the experimental rats, thereby reducing their stress $(16,17)$ (Fig. 2). Stainless steel needles were inserted at ST-36 and GB-34 at a depth of 2-3 $\mathrm{mm}$. A recent study indicated that alternating stimulation at low $(2 \mathrm{~Hz})$ and high $(100 \mathrm{~Hz})$ frequencies (referred to as 2/100 EA) elicits a synergistic analgesic effect (18). The intensity of EA was determined by observing slight shrinkage in the muscle, which was $\sim 1.5 \mathrm{~mA}$. Therefore, stimulation (current of 2/100 Hz, $1.5 \mathrm{~mA}$ ) was delivered using an electrical stimulation device (HANS-200E; Jisheng Medical Instruments, Nanjing, China) for $30 \mathrm{~min}$ daily.

Immunofluorescence. On day 14, the rats were deeply anaesthetised as previously described and perfused via the aorta with $200 \mathrm{ml}$ of normal saline, followed by $250 \mathrm{ml}$ of $4 \%$ paraformaldehyde in $0.1 \mathrm{M}$ phosphate-buffered saline (PBS, $\mathrm{pH}$ 7.4). The L4-L6 segments of the spinal cord were excised and fixed. Paraffin sections (5 $\mu \mathrm{m}$ thick) were mounted on poly-L-lysine-coated slides for immunofluorescence. They were deparaffinised with xylene and rehydrated through a graded ethanol series. After washing, the sections were blocked with $3 \% \mathrm{H}_{2} \mathrm{O}_{2}$ for $10 \mathrm{~min}$ and treated with a sodium citrate buffer at $95^{\circ} \mathrm{C}$ for antigen retrieval for $20 \mathrm{~min}$. After blocking in $10 \%$ normal goat serum in $0.01 \mathrm{M}$ PBS with $0.3 \%$ Triton $\mathrm{X}-100$ for $1 \mathrm{~h}$ at room temperature, the sections were incubated overnight at $4{ }^{\circ} \mathrm{C}$ with rabbit anti-ionised calcium binding adaptor molecule 1 (Iba1) (a microglia marker, 1:200, 019-19741; Wako, Osaka, Japan) and sheep anti-BDNF (1:100, ab75040; Abcam, Cambridge, MA, USA). Following the primary antibody incubation, the sections were incubated for $30 \mathrm{~min}$ at $37^{\circ} \mathrm{C}$ with the following secondary antibodies: fluorescein (FITC)-conjugated AffiniPure goat anti-rabbit IgG (1:200, BS10950; Bioworld, Minneapolis, MN, USA) and DyLight 594-labelled donkey anti-sheep IgG (1:200, ab96941; Abcam). The sections were subsequently stained with diamidino-2-phenylindole dihydrochloride (DAPI) $(1: 1,000$; Beyotime Corp., Shanghai, China). Finally, the sections were washed with PBS, and coverslips were mounted onto the slides using antifade mounting medium (Beyotime Corp.). The specific distributions of BDNF and Ibal expression within 
the spinal cord sections were determined with a fluorescence microscope (Olympus, Tokyo, Japan).

Quantitative real-time PCR. At the end of EA treatment, 5 randomly-selected rats from each group were deeply anesthetised with $4 \%$ chloral hydrate and perfused via the aorta with $400 \mathrm{ml}$ of normal saline. The lower lumbar enlargement (L4-6) region of the spinal cord was separated. Total RNA $(1 \mu \mathrm{g})$ was extracted from the tissue using TRIzol reagent (Invitrogen, Carlsbad, CA, USA). First-Strand cDNA was synthesised by a reverse transcriptase kit (A3500; Promega, Madison, WI, USA) according to the manufacturer's instructions. Real-time amplification, using SYBR-Green Supermix (QPK-212; Toyobo Corporation, Osaka, Japan) and a LightCycler 480 system (Roche, Indianapolis, IN, USA), was performed using the following sequences: Iba1, sense, GGATGGGATCAACAAG CACT and antisense, TCCATTGCCATTCAGATCAA; BDNF, sense, CTTGGAGAAGGAAACCGCCT and antisense, GTCC ACACAAAGCTCTCGGA; TrkB, sense, GCTTCTGGAGGG CTTCTCTT and antisense, TGTTCTCTGGGTCAATGCTG; and RPS16, sense, AAGTCTTCGGACGCAAGAAA and antisense, TTGCCCAGAAGCAGAACAG. The qPCR conditions were as follows: $95^{\circ} \mathrm{C}$ for $5 \mathrm{~min}$, followed by 40 cycles of $95^{\circ} \mathrm{C}$ for $10 \mathrm{sec}, 60^{\circ} \mathrm{C}$ for $10 \mathrm{sec}$, and $72^{\circ} \mathrm{C}$ for $10 \mathrm{sec}$. All samples were performed in triplicate. RPS16 was used as an internal control. The relative expression levels were analysed using the $2^{-\Delta \Delta \mathrm{Ct}}$ method with the relative expression software tool (19).

Western blotting. The rats were deeply anesthetised as previously described on day 14 after the CCI operation. A laminectomy was rapidly performed to expose the lumbar spinal cord, and the L4-L6 segment was excised. The segments were immediately stored at $-80^{\circ} \mathrm{C}$ for further analysis. The cell lysates were lysed in fresh RIPA protein lysis buffer that contained (RIPA: PMSF=100:1) on ice. The samples were subsequently incubated for $30 \mathrm{~min}$ and centrifuged at 15,294 $\mathrm{x} \mathrm{g}$ for $5 \mathrm{~min}$ at $4^{\circ} \mathrm{C}$, and the supernatant was collected. A BCA protein assay kit (Beyotime Corp.) was used to determine the protein concentration of each sample. The homogenate was heated to $100^{\circ} \mathrm{C}$ for $10 \mathrm{~min}$ and centrifuged again at $15,294 \mathrm{x} \mathrm{g}$ for $1 \mathrm{~min}$. Equal amounts $(80 \mu \mathrm{g})$ of protein were subjected to 8 and $12 \%$ Tris- $\mathrm{HCl}$ sodium dodecyl sulfate-polyacrylamide gel electrophoresis (SDS-PAGE) gel (Bio-Rad Laboratories, Hercules, CA, USA) separation for $30 \mathrm{~min}$ at $70 \mathrm{~V}$ and $60 \mathrm{~min}$ at $120 \mathrm{~V}$. After electrophoresis, the proteins were transferred onto PVDF membranes (Millipore Corp., Billerica, MA, USA) at $300 \mathrm{~mA}$ for immunoblotting. After blocking with $5 \%$ skim milk for $2 \mathrm{~h}$ at room temperature, the membranes were incubated overnight at $4^{\circ} \mathrm{C}$ with the following primary antibodies: rabbit anti-BDNF (1:1,000, ab108319; Abcam); rabbit anti-TrkB (phospho-Tyr705) (1:1,000, orb106262; Biorbyt, Cambridge, UK); rabbit anti-TrkB (1:700, ab33655; Abcam); and rabbit anti-glyceraldehyde-3-phosphate dehydrogenase (GAPDH) (1:5,000, AP0063; Bioworld). After three washes in TBST, the membranes were subsequently incubated with a horseradish peroxidase-conjugated goat anti-rabbit IgG (1:5,000, BL003A; Biosharp, St. Louis, MO, USA) for $2 \mathrm{~h}$ at room temperature. Protein bands were visualised using an enhanced chemiluminescence (ECL) kit (Beyotime Corp.). The band density was quantified via detection with a DNR micro-
$\mathbf{A}$
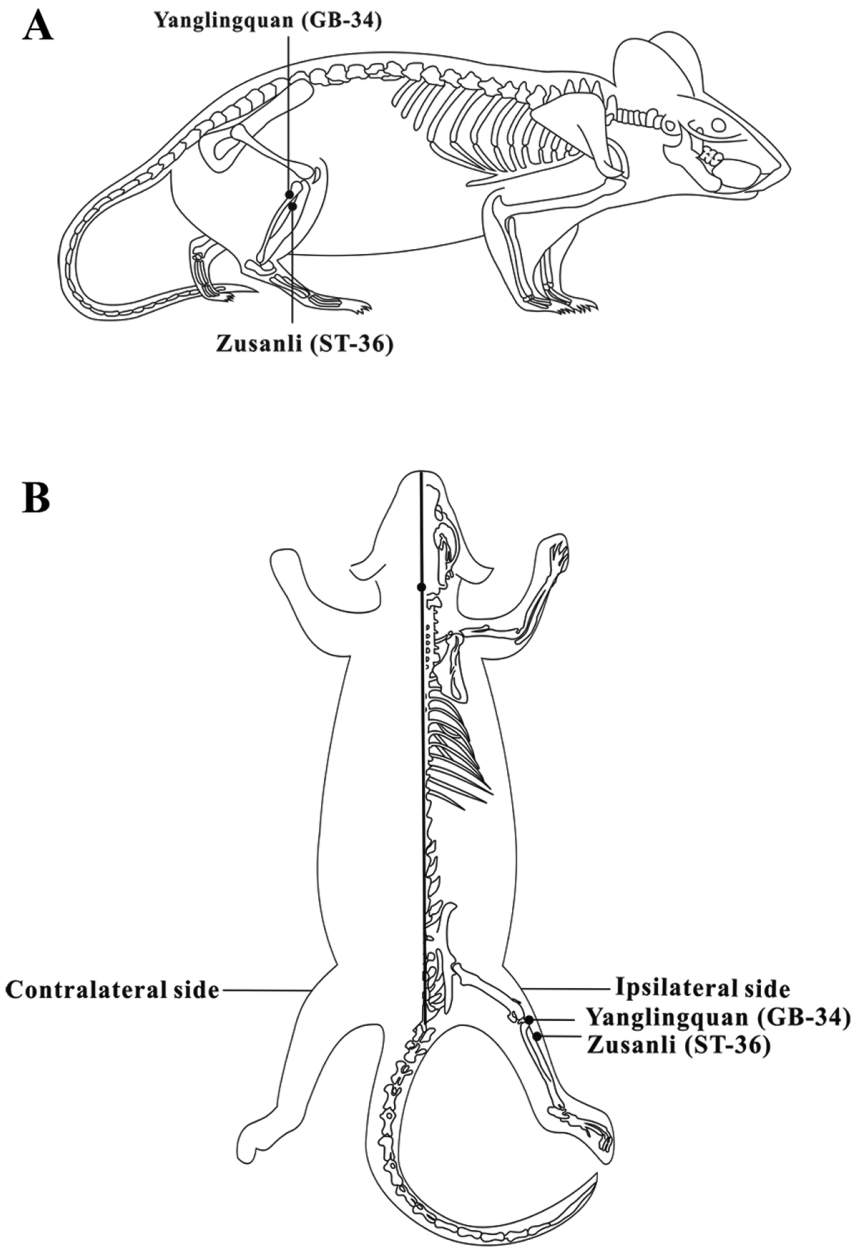

Figure 1. (A and B) Showed the locations of the 'Zusanli' (ST-36) and 'Yanglingquan' (GB-34) acupoints.

chemiluminescence gel imaging system (DNR Bio-Imaging Systems, Jerusalem, Israel). Moreover, each band density was normalised to the density of GAPDH.

Statistical analysis. SPSS 16.0 software was used for the statistical analysis. A statistical evaluation of the data was performed using a one-way analysis of variance (ANOVA) $(\mathrm{P}<0.05$, $\mathrm{P}<0.01$ and $\mathrm{P}<0.001$, followed by post hoc comparisons using the LSD or Kruskal-Wallis method. Data from the mechanical allodynia and thermal hyperalgesia tests were analysed via repeated-measure tests. All experimental data are expressed as the mean \pm standard deviation (SD), and $\mathrm{P}<0.05$ indicates a statistically significant difference.

\section{Results}

EA treatment reduces mechanical allodynia and thermal hyperalgesia in CCI rats. In this study, we investigated the potential efficacy of EA treatments in a rat model of CCI with regard to neuropathic pain. To determine the time-course of the changes in hyperalgesia and allodynia, baseline measurements were obtained prior to surgery. The baseline measures of the MWT and TWL on both hind paws did not differ among the four groups. In the CCI group, the MWT was markedly reduced after surgery, from $30.6 \pm 1.15$ to $10.9 \pm 1.42 \mathrm{~g}$, compared 


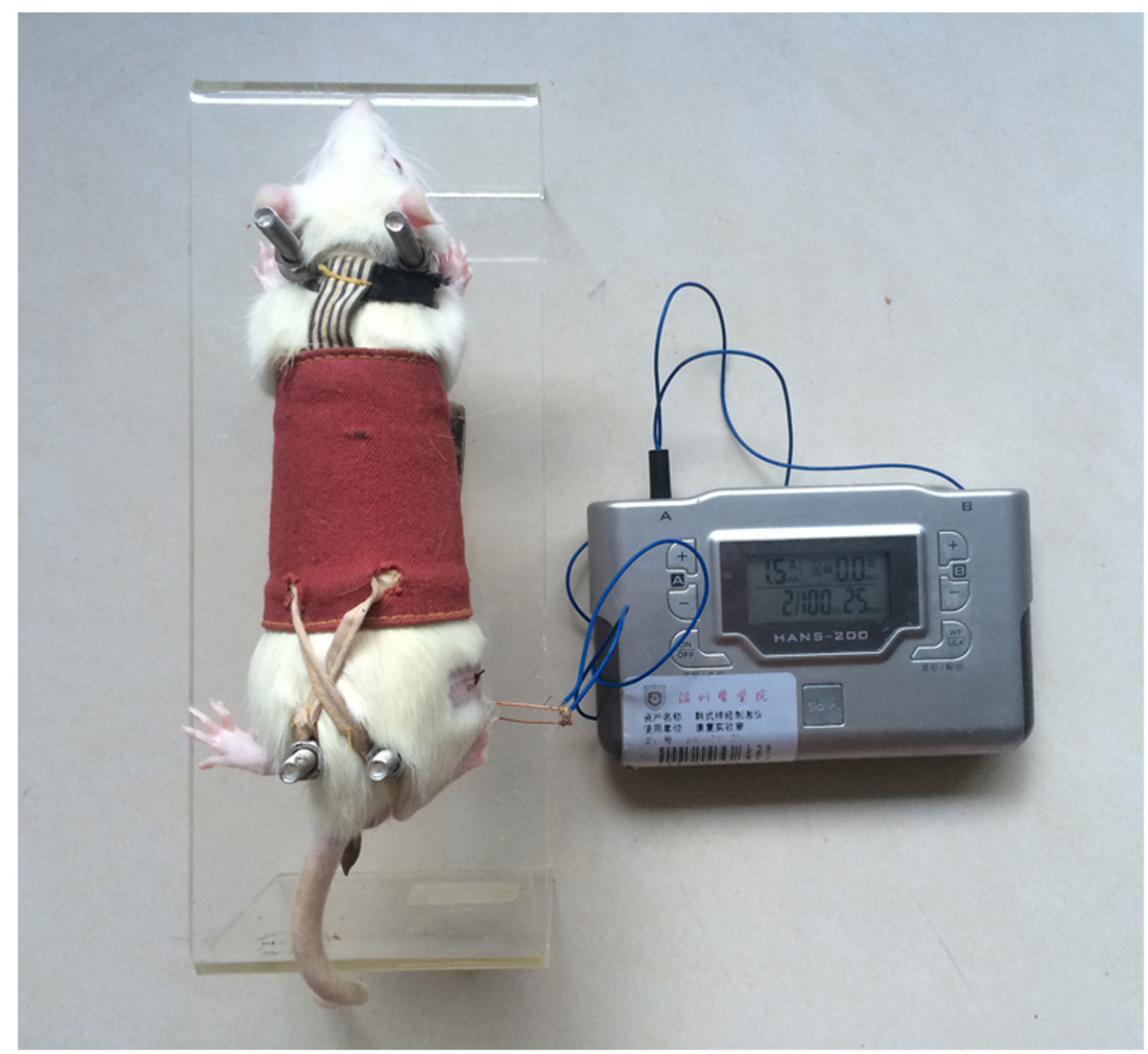

Figure 2. Rat immobilization apparatus designed by our laboratory was comfortable to experimental animals which can reduce stress and is very convenient for acupuncture research.
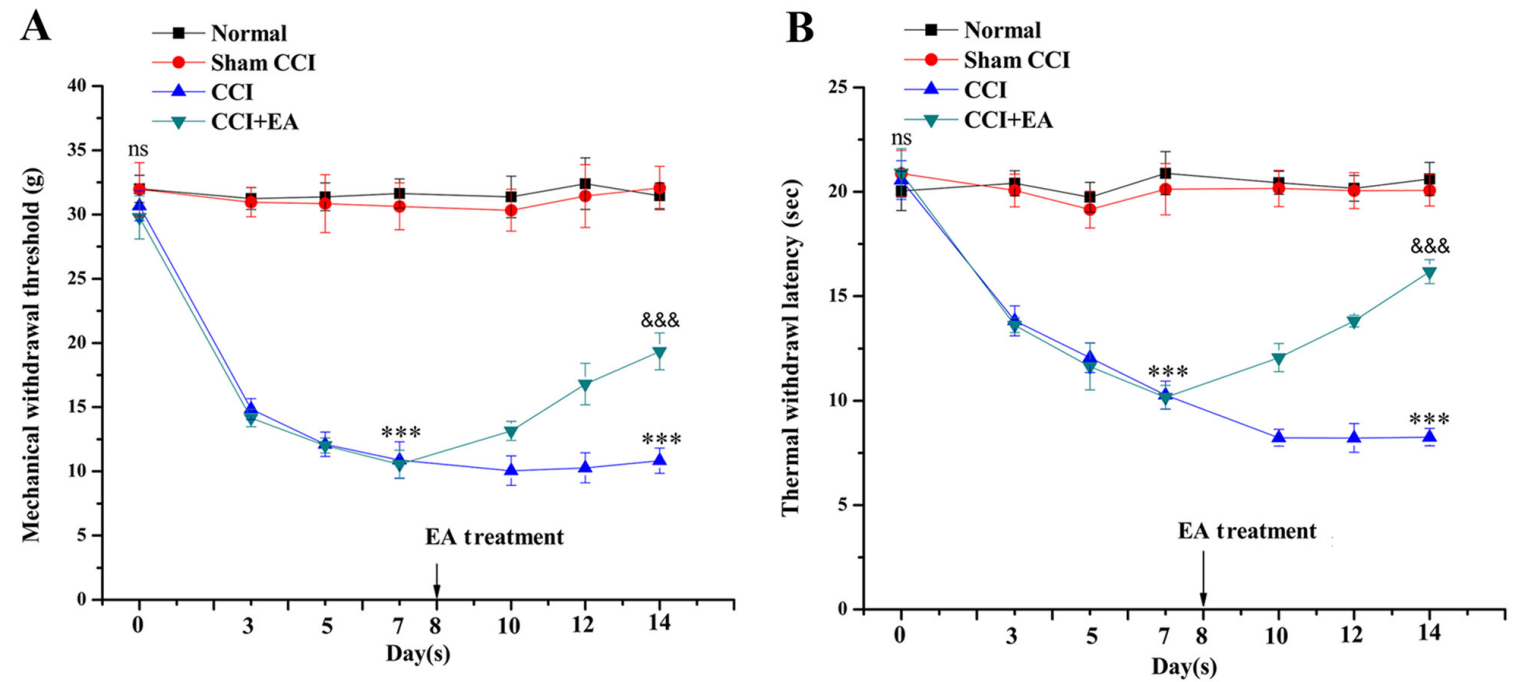

Figure 3. Analgesic effects of electro-acupuncture (EA) treatment on mechanical withdrawal threshold (MWT) and thermal withdrawal latency (TWL) in rats with chronic sciatic nerve constriction (n=15). The (A) MWT and (B) TWL in each group were recorded and compared among groups. There was no significance among the four groups before surgery; ${ }^{* * *} \mathrm{P}<0.001$ vs. the sham group; \&\&\& $\mathrm{P}<0.001$ vs. the chronic constrictive injury (CCI) group.

with the values in the normal and sham groups $(\mathrm{P}<0.001)$. The TWL was reduced from $21.9 \pm 0.87$ to $10.2 \pm 0.67 \mathrm{sec}$ on day $7(\mathrm{P}<0.001)$. On day 14, the MWT and TWL values of the CCI+EA group were significantly increased compared with the CCI group $(\mathrm{P}<0.001)$. These findings imply that EA treatment increases the mechanical and thermal pain thresholds in rats suffering from neuropathic pain following CCI surgery (Fig. 3).

Inhibitory effect of EA on the activation of microglia and $B D N F$ expression in the spinal cord of CCI rats demonstrated by immunofluorescence. The activation of microglia and BDNF 
A

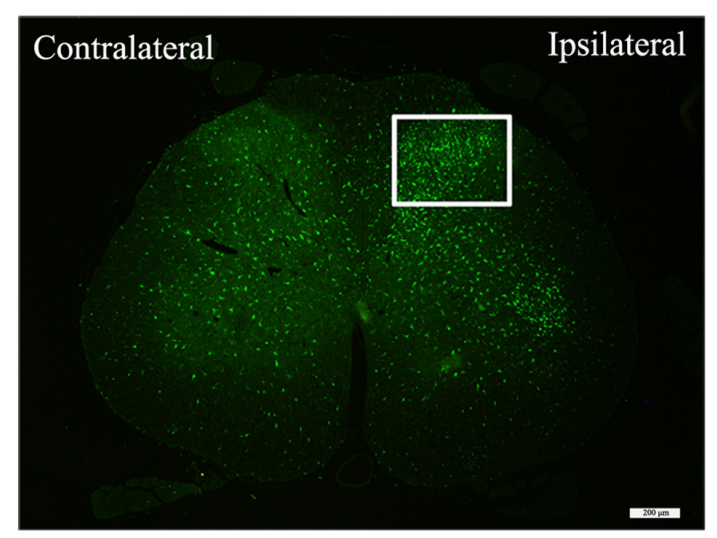

C
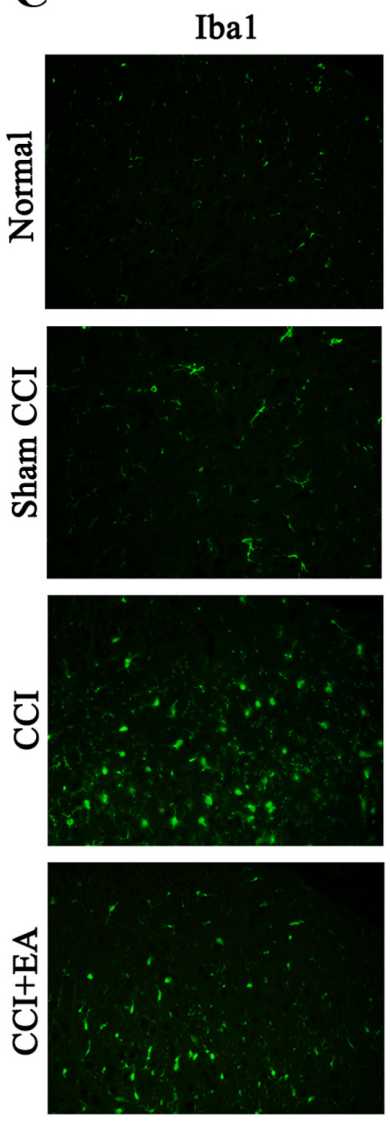

B

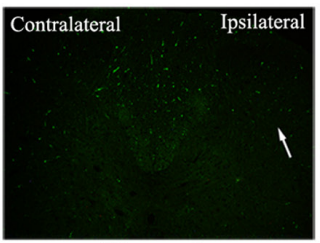

Normal

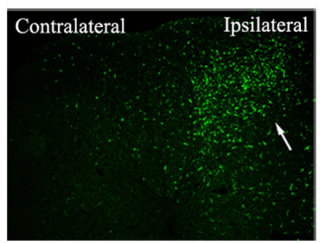

CCI
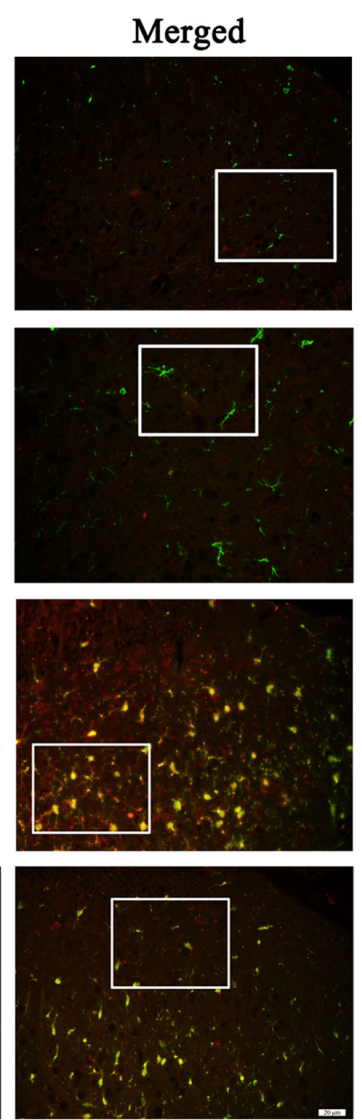

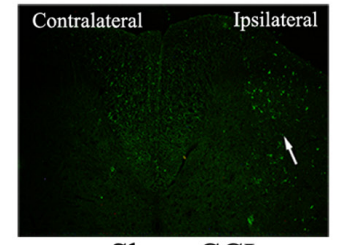

Sham CCI

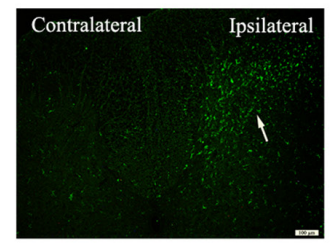

$\mathrm{CCI}+\mathrm{EA}$
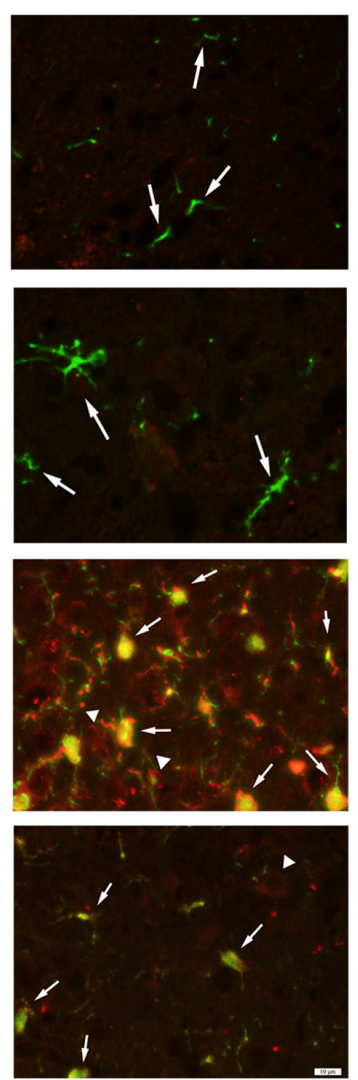

Figure 4. Chronic constrictive injury (CCI) induces microglia activation subsequent to the release of microglia brain-derived neurotrophic factor (BDNF). (A) White rectangle was the dorsal horn of the injured side of the spinal cord, immunofluorescence showed the amount of ionised calcium binding adaptor molecule 1 ionised calcium binding adaptor molecule 1 (Iba1) in the injured ipsilateral side relative to the contralateral, Iba1 was the marker for microglia. Scale bar, $200 \mu \mathrm{m}$. (B) Compare the expression of Iba1 in the injury ipsilateral side among the four groups, Scale bar, $100 \mu \mathrm{m}$. (C) Representative photomicrographs of immunofluorescence showed colocalization of Ibal (green, the arrows stand for Ibal ${ }^{+}$cells, represent activated microglia) and BDNF (red, the arrows represent $\mathrm{BDNF}^{+}$cells released from activated microglia, the white triangle represent the BDNF expressed in neurons in the spinal dorsal horns). Scale bar, $20 \mu \mathrm{m}$. The right side of the column; scale bar, $10 \mu \mathrm{m}$.

expression in the L4-L6 segment of the spinal dorsal horn was assessed via immunofluorescence. An increased number of activated microglia was identified accompanied by an upregulated expression of BDNF, which was mainly expressed in microglia. In addition, we also identified an increased level of BDNF in the neurons of the CCI group, which was released by activated microglia. However, CCI-induced activation of microglia and a high expression of BDNF were significantly inhibited by EA treatment (Fig. 4).
EA treatment downregulates spinal microglia activation and BDNF expression at the $m R N A$ and protein levels. To assess the role of microglia and BDNF in the spinal cord, qPCR analyses of microglia and BDNF were performed in each group. The qPCR analysis of the rats with CCI-induced neuropathic pain indicated significant decrease in the levels of microglia and BDNF in the CCI+EA rats compared with the $\mathrm{CCI}$ rats $(\mathrm{P}<0.001)$ (Fig. 5A and $\mathrm{B})$. The BDNF expression at the protein level was analysed via western blotting, which 
A

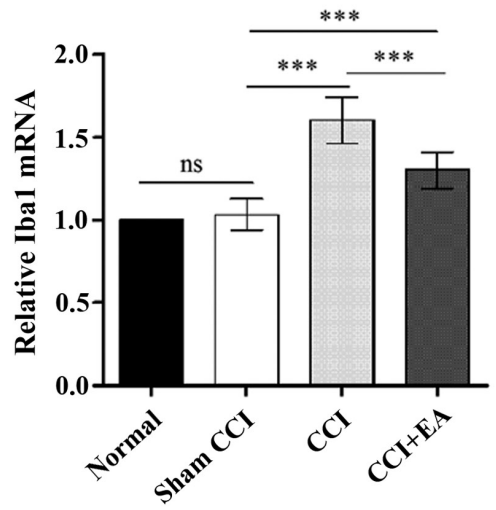

C

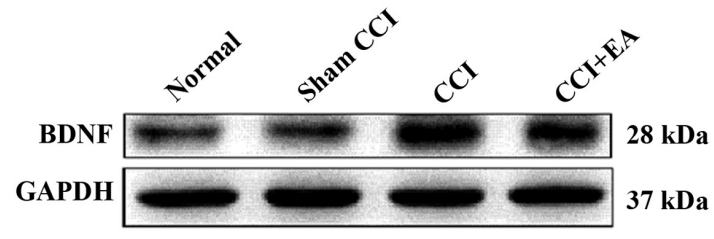

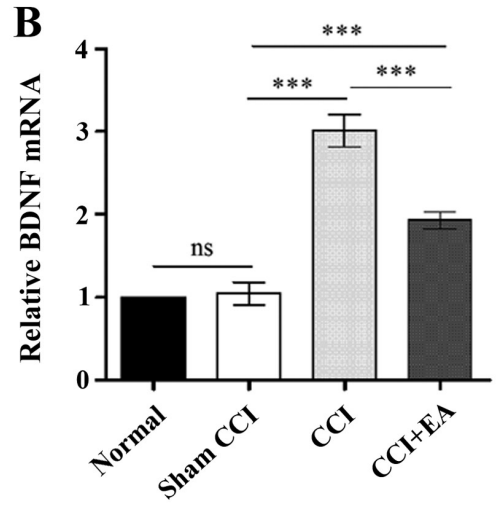

D

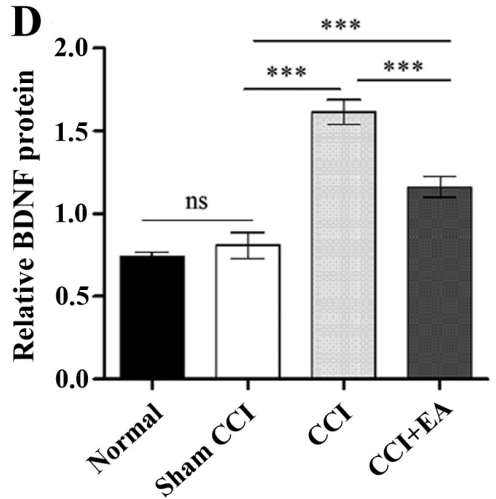

Figure 5. Increased ionised calcium binding adaptor molecule 1 (Iba1) and brain-derived neurotrophic factor (BDNF) expression in the spinal cord of chronic constrictive injury (CCI) rats. (A) The qPCR results showed the expression of Iba1 mRNA in the spinal cord. (B) The qPCR results showed the expression of BDNF mRNA in the spinal cord. (C) Protein expression of BDNF in the spinal cord segments. Glyceraldehyde-3-phosphate dehydrogenase (GAPDH) was used as the loading control and for band density normalization. (D) Optical density analysis of BDNF proteins. Values are expressed as the mean \pm SD, $n=5 / g r o u p$. ${ }^{* * * *} \mathrm{P}<0.001$ vs. the sham group; ${ }^{* * *} \mathrm{P}<0.001$ vs. the CCI group.

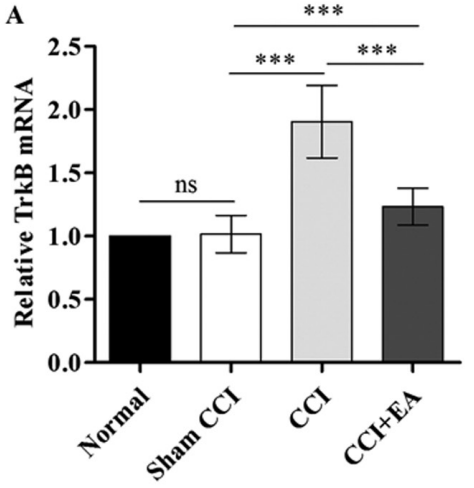

B

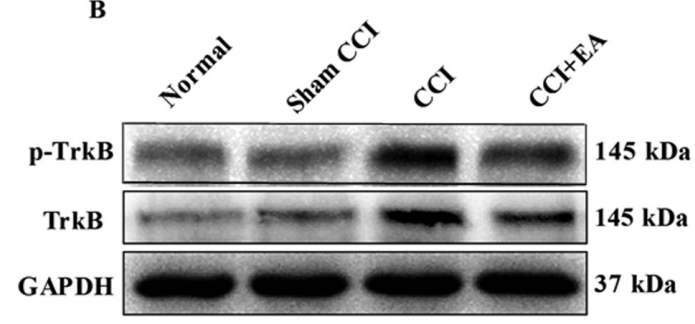

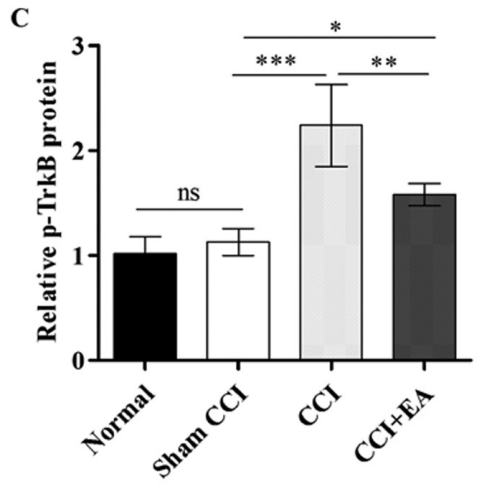

D

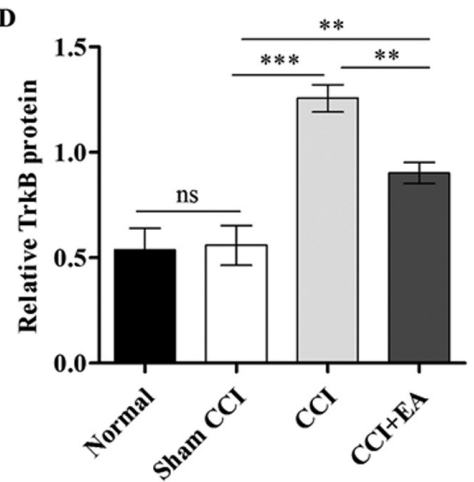

Figure 6. Effect of electro-acupuncture (EA) on the expression of tyrosine kinase receptor B (TrkB) in the spinal cord of chronic constrictive injury (CCI) rats. (A) The qPCR results showed the expression of TrkB mRNA in the spinal cord. (B) Protein expression of p-TrkB and TrkB in the spinal cord segments. Glyceraldehyde-3-phosphate dehydrogenase (GAPDH) was used as the loading control and for band density normalization. (C) Optical density analysis of p-TrkB proteins. (D) Optical density analysis of TrkB proteins. Values are expressed as the mean $\pm \mathrm{SD}, \mathrm{n}=5 /$ group. ${ }^{* * * *} \mathrm{P}<0.001$ vs. the sham group; ${ }^{* *} \mathrm{P}<0.05$ vs. the CCI group. 


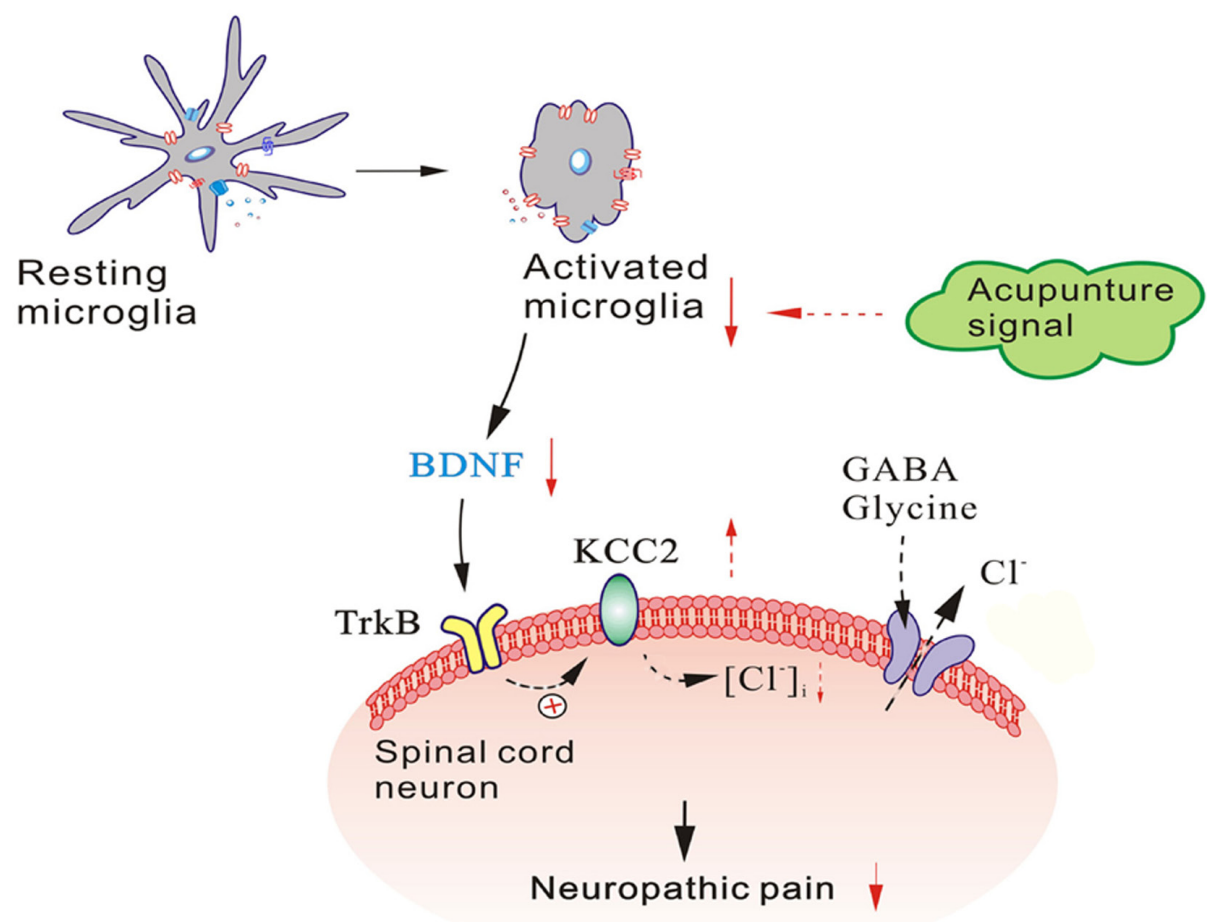

Figure 7. A schematic of the effect of electro-acupuncture on the brain-derived neurotrophic factor (BDNF)-tyrosine kinase receptor B (TrkB) pathway in the spinal cord. After peripheral nerve injury, spinal microglia could transform from a quiescent state into an activated state, which activated the BDNF-TrkB signalling pathway and maintained the pathophysiological processes. Electro-acupuncture (EA) could relieve neuropathic pain by inhibiting the activation of spinal microglia and disrupting the BDNF-TrkB signalling pathway.

indicated a strong increase in BDNF after the CCI operation. The relative optical density (ROD) value for the BDNF protein expression in the CCI group was significantly increased compared with in the normal, sham CCI and EA groups $(\mathrm{P}<0.001)$ (Fig. 5C and D), and EA treatment decreased the BDNF expression in the EA group. Taken together, these findings suggest a direct link between the effects of EA treatment and microglia activation and BDNF expression in CCI rats.

EA attenuates the overexpression of TrkB at both the $m R N A$ and protein levels. A substantial decrease in TrkB mRNA expression was identified in the spinal cord after EA relative to the expression in the CCI rats $(\mathrm{P}<0.001)$ (Fig. 6A). The TrkB expression at the protein level in the spinal cord was analysed via western blotting. The ROD for the $\mathrm{p}-\mathrm{TrkB}$ and TrkB protein expression in the CCI group were significantly increased compared with the normal and sham CCI groups $(\mathrm{P}<0.001)$. Similar to the results of $\mathrm{qPCR}$ analysis, the level of p-TrkB and TrkB protein was significantly decreased after EA treatment $(\mathrm{P}<0.01)$ (Fig. 6B-D). These findings indicate that EA significantly inhibits the expression of TrkB at both the mRNA and protein levels.

\section{Discussion}

Acupuncture has been used for more than 3,000 years in China and is generally regarded as a safe and effective method to alleviate pain in humans and experimental animals (20-23). EA treatments apply various levels of stimulating currents to acupoints through acupuncture needles. EA is widely practiced worldwide; however, the biological basis of the analgesic effects of EA on neuropathic pain remains unclear. Various neurotransmitters and neuromodulators, including primarily opioid peptides, glutamate, norepinephrine, serotonin, g-amino-butyric acid and adenosine have been suggested to be responsible for the beneficial effects of EA on neuropathic pain $(23,24)$.

CCI rats have been demonstrated to exhibit behavioural signs of spontaneous pain and hyperalgesia as a result of noxious thermal and mechanical stimuli (23). In this study, mechanical allodynia and thermal hyperalgesia persisted in the CCI group; however, all rats in the group began to exhibit signs of pain relief after 7 days of EA treatment at the ST-36 and GB-34 acupoints. This finding indicates acupuncture may provide an effective treatment for pain and has potential in clinical applications.

One of the most significant advances in pain research was the finding that neurons are not the only cell type involved in the aetiology of chronic pain. This discovery caused a radical shift from the previous dogma that neuronal dysfunction alone accounted for pain pathologies to a novel type of thinking, in which all cell types within the CNS should be considered with respect to their involvement in neuropathic pain (25). In recent years, additional studies focused on this topic have suggested a significant role of microglia in the maintenance of normal neuronal physiology in the CNS. The activation of microglia has been associated with symptoms of neuropathic pain such as allodynia or hyperalgesia (26). Accumulated evidence suggests that spinal microglia are involved in the modulation of chronic pain and EA analgesia $(27,28)$. Choi et al demonstrated that acupuncture not only relieves mechanical allodynia and thermal hyperalgesia but also decreases the proportion of acti- 
vated microglia at the L4-5 when applied at the Shuigou (GV26) and Yanglingquan (GB-34) acupoints following SCI-induced neuropathic pain (29). Shan et al provided the first indication that repeated EA significantly blocks the activation of spinal microglia and reduces the release of proinflammatory cytokines and other pain-enhancing substances in the spinal cord, which suggests that the anti-allodynic and anti-hyperalgesic effects of EA may be associated with its capacity to inhibit spinal microglia activation (30).

Ionised calcium binding adaptor molecule 1 (Iba1) comprises a sensitive marker associated with activated microglia (31). In this study, we demonstrated that EA treatment increased the MWT and TWL values. Furthermore, our immunofluorescence-based analysis indicated that the CCI-induced activation of microglia was significantly inhibited by EA treatment.

Activated microglia secrete various biologically active signalling molecules, including BDNF, which is a crucial molecule for signalling between microglia and neurons. The inhibition of this microglia-neuron signalling pathway may prevent tactile allodynia (32). Many studies have indicated that microglia are closely associated with the actions of BDNF; thus, the stimulation of BDNF release appears to be caused by activated microglia. These actions contributed to the establishment and maintenance of central sensitisation in chronic neuropathic pain. The present data illustrate that the proportion of activated microglia and the BDNF protein level were both increased in the spinal dorsal horn in association with pathological pain. However, EA significantly reversed this phenomenon by decreasing the activation of microglia and BDNF expression, thereby attenuating the hyperalgesia and allodynia associated with neuropathic pain.

Furthermore, recent studies have indicated that BDNF exerts its effect by binding to its high affinity receptor TrkB, and that the BDNF-TrkB pathway may be an important early step in the transition from normal to pathophysiological processing in the spinal dorsal horn (33). Both in vivo and in vitro studies, have demonstrated that the activation of the BDNF-TrkB pathway leads to a downregulation of potassium-chloride co-transporter (KCC2) expression in neurons (32,34-36). The expression of $\mathrm{KCC} 2$, the main $\mathrm{Cl}^{-}$transporter in spinal lamina I neurons, is downregulated by BDNF, which causes an increase in intracellular $\left[\mathrm{Cl}^{-}\right]$within these cells (35). As a result, the opening of $\gamma$-amino butyric acid (GABA)A or glycine channels becomes less effective in producing inhibition, and in approximately one-third of lamina I neurons, GABA-evoked responses are converted from hyperpolarising to depolarising responses $(32,37,38)$.

In this study, the expression of TrkB in the L4-L6 segment was examined via western blotting and qPCR analysis, which demonstrated that EA treatment decreased expression of $\mathrm{p}$-TrkB and TrkB receptors. These data further support the notion that BDNF-TrkB signalling pathway interactions mediated by spinal microglia play a crucial role in the development of the hyperalgesia and allodynia associated with chronic pain. Based on these findings, we suspect that EA may relieve neuropathic pain via the regulation of this signalling pathway (Fig. 7). In future studies, we intend to apply pharmacological, gene knock-out and other methods to further test and verify this hypothesis.
In conclusion, we demonstrated that mechanical allodynia and thermal hyperalgesia appear to be attenuated by EA via its analgesia effects. Excessive spinal microglia activation induced by nerve injury, which activates the BDNF-TrkB signalling pathway and maintains the pathophysiological processes that lead to neuropathic pain, is substantially inhibited by EA. However, further verification of the overall effects and the underlying molecular mechanism of EA in animals with peripheral nerve injuries is necessary.

\section{Acknowledgements}

The experiments were carried out with the help of the Scientific Research Center, The Second Affiliated Hospital of Wenzhou Medical University.

\section{Funding}

This study was supported by a grant from the Natural Science Foundation of Zhejiang Province (LY16H270016) and the Foundation of Wenzhou Scientific and Technological Bureau Project (Y20140221).

\section{Availability of data and material}

The datasets used and/or analyzed during the current study are available from the corresponding author on reasonable request.

\section{Authors' contributions}

WZT, SHJ and conceived and designed the experiments. SSL and XJ performed the experiments. XRQ, GHY and PPG analyzed the data and created the images. WZT and SSL wrote the paper. BL and SHJ contributed to the modification of the manuscript. All authors read and approved the final manuscript.

\section{Ethics approval and consent to participate}

The Institutional Animal Care and Use Committee of Wenzhou Medical University approved all experiments, which were performed according to the guidelines of the National Institutes of Health Guide for the Care and Use of Laboratory Animals (NIH Publications no. 80-23, revised 1978).

\section{Consent for publication}

Not applicable.

\section{Competing interests}

The authors declare that they have no competing interests.

\section{References}

1. Baron R: Neuropathic pain: A clinical perspective. Handb Exp Pharmacol 194: 3-30, 2009.

2. Goettl VM, Huang Y, Hackshaw KV and Stephens RL Jr: Reduced basal release of serotonin from the ventrobasal thalamus of the rat in a model of neuropathic pain. Pain 99: 359-366, 2002

3. Smith HS: Opioids and neuropathic pain. Pain Physician 15 (Suppl 3): ES93-ES110, 2012 
4. Ren W, Tu W, Jiang S, Cheng R and Du Y: Electroacupuncture improves neuropathic pain: Adenosine, adenosine 5'-triphosphate disodium and their receptors perhaps change simultaneously. Neural Regen Res 7: 2618-2623, 2012.

5. Tu W, Wang W, Xi H, He R, Gao L and Jiang S: Regulation of neurotrophin-3 and interleukin-1beta and inhibition of spinal glial activation contribute to the analgesic effect of electroacupuncture in chronic neuropathic pain states of rats. Evid Based Complement Alternat Med 2015: 642081, 2015.

6. Tsuda M, Beggs S, Salter MW and Inoue K: Microglia and intractable chronic pain. Glia 61: 55-61, 2013.

7. Zhang X, Xu Y, Wang J, Zhou Q, Pu S, Jiang W and Du D: The effect of intrathecal administration of glial activation inhibitors on dorsal horn BDNF overexpression and hind paw mechanical allodynia in spinal nerve ligated rats. J Neural Transm Vienna 119: 329-336, 2012.

8. Pandya CD, Kutiyanawalla A and Pillai A: BDNF-TrkB signaling and neuroprotection in schizophrenia. Asian J Psychiatr 6: 22-28, 2013.

9. Xing GG, Liu FY, Qu XX, Han JS and Wan Y: Long-term synaptic plasticity in the spinal dorsal horn and its modulation by electroacupuncture in rats with neuropathic pain. Exp Neurol 208: 323-332, 2007

10. Kim SK, Park JH, Bae SJ, Kim JH, Hwang BG, Min BI, Park DS and Na HS: Effects of electroacupuncture on cold allodynia in a rat model of neuropathic pain: Mediation by spinal adrenergic and serotonergic receptors. Exp Neurol 195: 430-436, 2005.

11. Lau WK, Lau YM, Zhang HQ, Wong SC and Bian ZX: Electroacupuncture versus celecoxib for neuropathic pain in rat SNL model. Neuroscience 170: 655-661, 2010.

12. Sun S, Cao H, Han M, Li TT, Zhao ZQ and Zhang YQ: Evidence for suppression of electroacupuncture on spinal glial activation and behavioral hypersensitivity in a rat model of monoarthritis. Brain Res Bull 75: 83-93, 2008.

13. Fox A, Kesingland A, Gentry C, McNair K, Patel S, Urban L and James I: The role of central and peripheral Cannabinoid1 receptors in the antihyperalgesic activity of cannabinoids in a model of neuropathic pain. Pain 92: 91-100, 2001.

14. Huang C, Li HT, Shi YS, Han JS and Wan Y: Ketamine potentiates the effect of electroacupuncture on mechanical allodynia in a rat model of neuropathic pain. Neurosci Lett 368: 327-331, 2004

15. Gao YH, Wang JY, Qiao LN, Chen SP, Tan LH, Xu QL and Liu JL: NK cells mediate the cumulative analgesic effect of electroacupuncture in a rat model of neuropathic pain. BMC Complement Altern Med 14: 316, 2014

16. Tu WZ, Cheng RD, Cheng B, Lu J, Cao F, Lin HY, Jiang YX, Wang JZ, Chen $\mathrm{H}$ and Jiang SH: Analgesic effect of electroacupuncture on chronic neuropathic pain mediated by $\mathrm{P} 2 \mathrm{X} 3$ receptors in rat dorsal root ganglion neurons. Neurochem Int 60 : 379-386, 2012.

17. Wang WS, Tu WZ, Cheng RD, He R, Ruan LH, Zhang L, Gong YS, Fan XF, Hu J, Cheng B, et al: Electroacupuncture and A-317491 depress the transmission of pain on primary afferent mediated by the $\mathrm{P} 2 \mathrm{X} 3$ receptor in rats with chronic neuropathic pain states. J Neurosci Res 92: 1703-1713, 2014.

18. Silva JR, Silva ML and Prado WA: Analgesia induced by 2- or $100-\mathrm{Hz}$ electroacupuncture in the rat tail-flick test depends on the activation of different descending pain inhibitory mechanisms. J Pain 12: 51-60, 2011.

19. Livak KJ and Schmittgen TD: Analysis of relative gene expression data using real-time quantitative PCR and the 2(-Delta Delta C(T)) method. Methods 25: 402-408, 2001.

20. Filshie J: The non-drug treatment of neuralgic and neuropathic pain of malignancy. Cancer Surv 7: 161-193, 1988.

21. Wong JY and Rapson LM: Acupuncture in the management of pain of musculoskeletal and neurologic origin. Phys Med Rehabil Clin N Am 10: 531-545, vii-viii, 1999.
22. Qin Z, Liu X, Yao Q, Zhai Y and Liu Z: Acupuncture for treating sciatica: A systematic review protocol. BMJ Open 5: e007498, 2015.

23. Zhao ZQ: Neural mechanism underlying acupuncture analgesia. Prog Neurobiol 85: 355-375, 2008.

24. Chen S, Wang S, Rong P, Wang J, Qiao L, Feng X, Liu J and Zhang J: Acupuncture for visceral pain: neural substrates and potential mechanisms. Evid Based Complement Alternat Med 2014: 609594, 2014.

25. Trang T, Beggs S and Salter MW: Brain-derived neurotrophic factor from microglia: A molecular substrate for neuropathic pain. Neuron Glia Biol 7: 99-108, 2011.

26. Mika J, Zychowska M, Popiolek-Barczyk K, Rojewska E and Przewlocka B: Importance of glial activation in neuropathic pain. Eur J Pharmacol 716: 106-119, 2013.

27. Inoue $\mathrm{K}$, Tsuda $\mathrm{M}$ and Tozaki-Saitoh $\mathrm{H}$ : Role of the glia in neuropathic pain caused by peripheral nerve injury. Brain Nerve 64: 1233-1239, 2012 (In Japanese).

28. Liang LL, Yang JL, Lü N, Gu XY, Zhang YQ and Zhao ZQ: Synergetic analgesia of propentofylline and electroacupuncture by interrupting spinal glial function in rats. Neurochem Res 35 : 1780-1786, 2010.

29. Choi DC, Lee JY, Lim EJ, Baik HH, Oh TH and Yune TY: Inhibition of ROS-induced p38MAPK and ERK activation in microglia by acupuncture relieves neuropathic pain after spinal cord injury in rats. Exp Neurol 236: 268-282, 2012.

30. Shan S, Qi-Liang MY, Hong C, Tingting L, Mei H, Haili P, Yan-Qing W, Zhi-Qi Z and Yu-Qiu Z: Is functional state of spinal microglia involved in the anti-allodynic and anti-hyperalgesic effects of electroacupuncture in rat model of monoarthritis? Neurobiol Dis 26: 558-568, 2007.

31. Narita M, Yoshida T, Nakajima M, Narita M, Miyatake M, Takagi T, Yajima Y and Suzuki T: Direct evidence for spinal cord microglia in the development of a neuropathic pain-like state in mice. J Neurochem 97: 1337-1348, 2006.

32. Coull JA, Beggs S, Boudreau D, Boivin D, Tsuda M, Inoue K, Gravel C, Salter MW and De Koninck Y: BDNF from microglia causes the shift in neuronal anion gradient underlying neuropathic pain. Nature 438: 1017-1021, 2005

33. Zhang W, Liu LY and Xu TL: Reduced potassium-chloride co-transporter expression in spinal cord dorsal horn neurons contributes to inflammatory pain hypersensitivity in rats. Neuroscience 152: 502-510, 2008

34. Rivera C, Voipio J, Thomas-Crusells J, Li H, Emri Z, Sipilä S, Payne JA, Minichiello L, Saarma M and Kaila K: Mechanism of activity-dependent downregulation of the neuron-specific $\mathrm{K}-\mathrm{Cl}$ cotransporter KCC2. J Neurosci 24: 4683-4691, 2004.

35. Coull JA, Boudreau D, Bachand K, Prescott SA, Nault F, Sík A, De Koninck P and De Koninck Y: Trans-synaptic shift in anion gradient in spinal lamina I neurons as a mechanism of neuropathic pain. Nature 424: 938-942, 2003.

36. Biggs JE, Lu VB, Stebbing MJ, Balasubramanyan S and Smith PA: Is BDNF sufficient for information transfer between microglia and dorsal horn neurons during the onset of central sensitization? Mol Pain 6: 44, 2010.

37. Kahle KT, Staley KJ, Nahed BV, Gamba G, Hebert SC, Lifton RP and Mount DB: Roles of the cation-chloride cotransporters in neurological disease. Nat Clin Pract Neurol 4: 490-503, 2008.

38. Cordero-Erausquin M, Coull JA, Boudreau D, Rolland M and De Koninck Y: Differential maturation of GABA action and anion reversal potential in spinal lamina I neurons: Impact of chloride extrusion capacity. J Neurosci 25: 9613-9623, 2005.

This work is licensed under a Creative Commons Attribution-NonCommercial-NoDerivatives 4.0 International (CC BY-NC-ND 4.0) License. 